\title{
Characteristics of stereomovement suppression
}

\author{
C. WILLIAM TYLER \\ Northeastern University, Boston, Massachusetts 02115
}

\begin{abstract}
The suppression of visibility of stereomovement was investigated for sinusoidal depth movement of a test line with an antiphase reference line. Suppression occurred above a rate of oscillation of $1 \mathrm{~Hz}$, whereas enhancement was observed below $1 \mathrm{~Hz}$. Suppression was also found for vertical movement of horizontal stimulus lines. Suppression must therefore result from the processes of fusion rather than stereoscopic depth perception. Suppression was reduced with peripheral observation of the stimulus.
\end{abstract}

A previous investigation (Tyler, 1971) established the existence of a stereomovement suppression effect. For a stereoscopically generated vertical-line stimulus, sensitivity to sinusoidal depth movement was reduced by about a factor of three in relation to the retinally equivalent monocular movement sensitivity. The suppression effect was found to occur only to the extent that fused depth movement was present in the stimulus.

Stereomovement suppression represents a significant limitation on the ability to process information about the human environment. For example, in seeing vehicles at night, binocular observation will result in suppression of monocularly available information that distant vehicles are in motion, in addition to the reduction of depth movement information. It is therefore important to study the range of conditions over which stereomovement suppression occurs. This paper describes the extent of stereomovement suppression under three conditions: (1) with an antiphase reference line to eliminate any possibility of vergent tracking of the stimulus; (2) with vertical movement of horizontal lines (if suppression occurs under these conditions, it must result from processing for fusion rather than depth, since depth is not perceived for vertical disparities); and (3) as a function of retinal eccentricity to establish the generality of suppression across the retina.

It is conceivable that in the previous study, despite fixation instructions, differential eye tracking in the monocular and stereoscopic conditions might have affected the results at low frequencies. The stimulus consisted of a sinusoidally moving test line and a stationary reference and fixation line. Suppose the subject had fixated the reference line in the monocular condition but had tracked the average stimulus movement with vergent eye movements in the stereoscopic condition. It would thus have been possible to reduce the retinal movement of the test line by a factor of 2 in the stereoscopic condition. with

Supported by the Foundations Fund for Research in Psychiatry, Grant 70-481. The author's present address is: Bell Laboratories, Murray Hill, New Jersey 07974. movement of the same amplitude now produced in the reference line. Both movements could then have been set to threshold, reducing the measured sensitivity by a factor of 2 . If the same effect did not occur in the monocular condition, this difference would account for part of the stereomovement suppression at low frequencies. Since the vergence eye-movement system is ineffective above about $2 \mathrm{~Hz}$ (Zuber \& Stark, 1968), the results should not be affected above this frequency.

\section{STEREOMOVEMENT SUPPRESSION WITH AN ANTIPHASE REFERENCE}

A stimulus in which both lines moved in antiphase would control for this possible eye-tracking difference. Sensitivity for movement in this type of stimulus was therefore measured at a range of frequencies.

\section{Method}

The display stimuli consisted of thin, bright vertical lines, subtending 1.5 arc deg in height and 10 arc min in width seen against a dark background. They were produced on the face of a fast-phosphor Dumont oscilloscope by a sawtooth signal at $30 \mathrm{kHz}$ with a luminance of $3.4 \mathrm{~cd} / \mathrm{m}^{2}$ and were viewed with a natural pupil, because there was no overall change in stimulus luminarice. The stimuli were viewed stereoscopically at a distance of $33 \mathrm{~cm}$ by means of the conventional arrangement of orthogonal polatizers. Convergence was adjusted to the same distance by means of prismatic lenses to prevent a mismatch between convergence and accommodation cues. These experimental conditions were very similar to those used previously (Tyler, 1971). The lines were displaced sinusoidally (i.e., in simple harmonic motion) with an amplitude that could be varied by the subject. The stimulus configuration observed by the subject consisted of two lines oscillating in depth in antiphase. Thus, one line moved forward as the other moved back, and vice versa. The mean separation between the lines was 20 arc min. Both subjects viewed the display with normal refractive correction.

Visual sensitivities to monocular and stereoscopic depth movement were compared as a function of oscillation frequency. The subject, who was instructed to fixate between the moving lines, set the movement amplitude of both lines until they appeared just not visibly moving. Peak-to-peak amplitude of movement at this threshold setting was taken as the reciprocal of sensitivity. Monocular movement amplitude was taken as the measure in the case of the stereoscopic movement, in order to make a comparison 

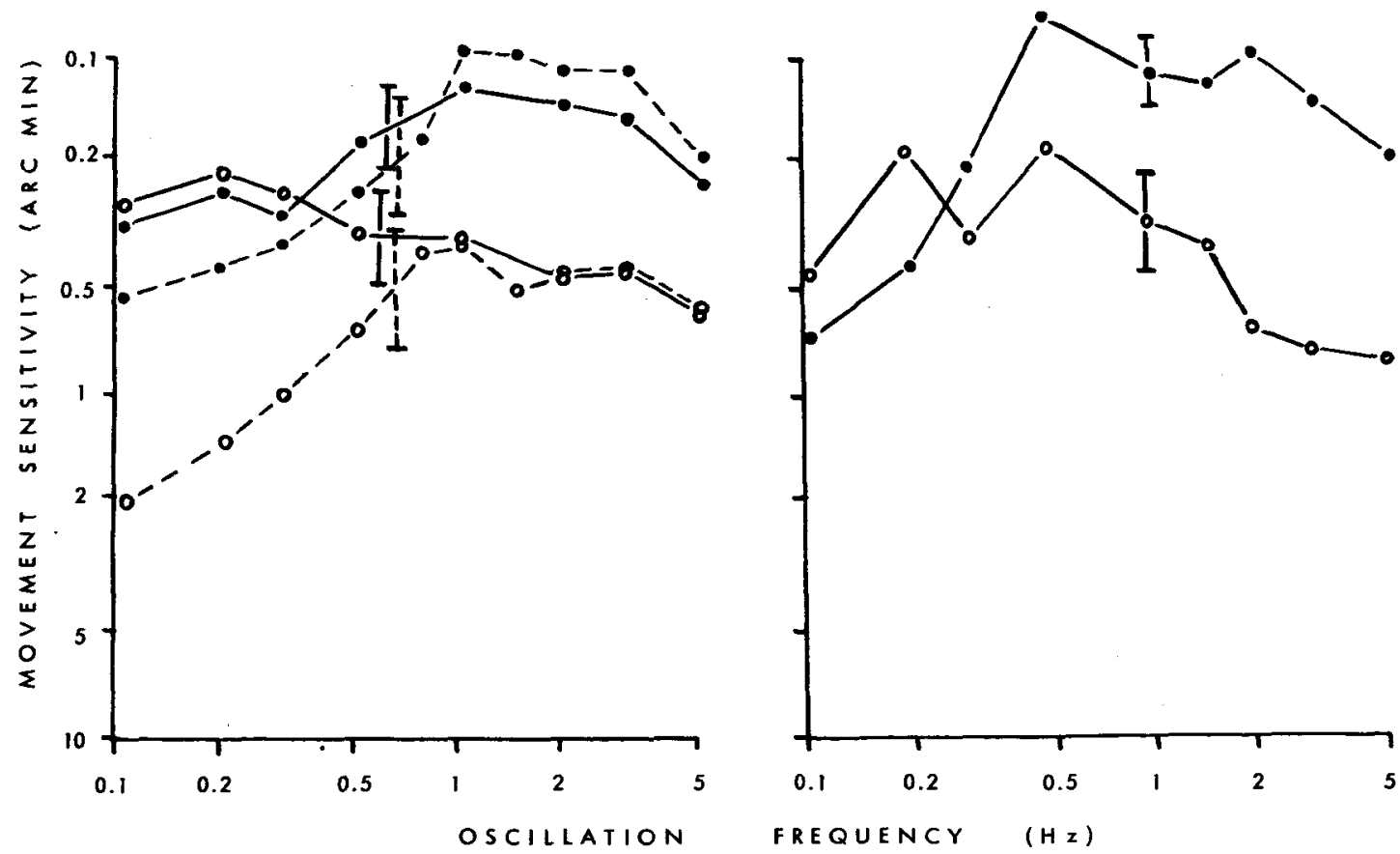

Figure 1. Stereomovement suppression with anttphase reference for C.W.T. (left-hand graph) and D.B. (right-hand graph). Full circles, monocular sensitivity; open circles, stereomovement sensittity; full limes, antiphase reference; dashed lines, stationary reference.

of monocular movement information required for monocular as compared to stereoscopic movement threshold. If the subject fixated between the two lines, average movement of the stimulus was zero. Any attempt to track one line would increase the amplitude of retinal movement of the other.

\section{Results}

Monocular and stereoscopic depth movement sensitivity for the antiphase stimulus as a function of frequency is plotted in Figure 1 for two subjects. Each point is the mean of two readings. The vertical bars represent one standard deviation on either side of the mean computed from all points in a given condition. Sensitivity for stereomovement differs significantly from that for monocular movement above $0.5 \mathrm{~Hz}$, but not below. Monocular and stereoscopic depth movement sensitivities for one subject are replotted from Tyler (1971) for comparison.

The frequency response data indicate that use of the antiphase reference stimulus (full lines, Figure 1) increased stereomovement sensitivity at low frequencies relative to sensitivity with the stationary reference stimulus (dashed lines, Figure 1). Monocular movement (full circles) sensitivity is not significantly affected.

\section{Discussion}

The initial question is whether vergence eye movements can account for the results, even though it is unlikely that vergence movements could occur over as little as 1 arc min. The increase in stereomovement sensitivity using the antiphase rather than stationary reference reaches almost a factor of 10 at $0.1 \mathrm{~Hz}$, whereas eye-movement tracking could only have produced a difference of a factor of 2. Furthermore, tracking ability for vergence eye movements in both gain and phase is approximately flat to $1 \mathrm{~Hz}$ with a steep decline thereafter (Zuber \& Stark, 1968; Yoshida \& Watanabe, 1969). In contrast, the gain characteristic required to account for the differences between stationary and antiphase reference lines would be a steady decrease in gain from $0.1 \mathrm{~Hz}$, reaching zero at $1 \mathrm{~Hz}$. For both these reasons, vergence eye movements may be considered a negligible factor in the results.

An alternative possibility is that the stationary/ antiphase reference difference represents an interaction between cortical disparity detectors. It has previously been suggested (Tyler, 1971) that there may be differential disparity inhibition between the detectors of different disparities at approximately the same retinal locations. Lateral disparity inhibition between detectors of the same disparity at different retinal locations was not postulated.

The stationary vs. antiphase reference conditions correspond to flicker studies using steady and antiphase flickering surrounds. Levinson (1964) and Kelly (1969) have shown that sensitivity to flicker is enhanced at low frequencies with antiphase flicker relative to a steady surround. This enhancement is attributed to lateral inhibition in the retina. A similar effect may occur with disparate stimuli. This may be expressed in terms of two disparity detectors, back and forward, at each retinal location (see Figure 2). If there is both lateral and differential disparity 


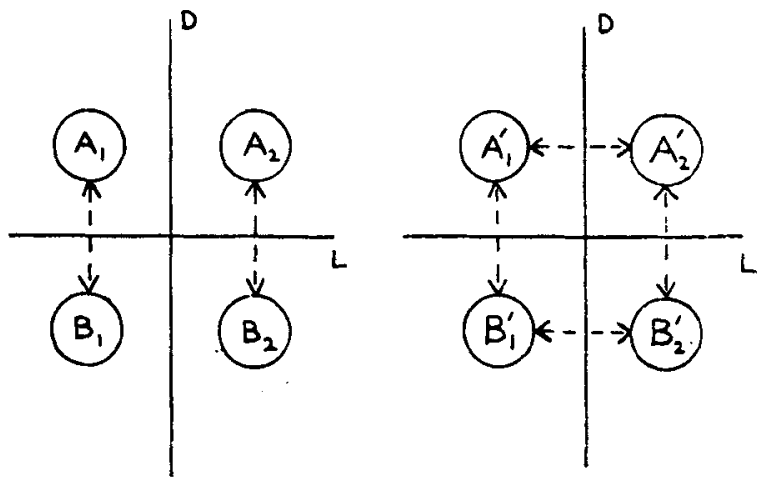

Figure 2. Model of types of disparity inhibition (see text for explanation). Inhibitory pathways shown as dashed lines, $L=$ lateral and D = depth plane in cyclopean space.

inhibition, then when the line at one retinal location is "back" it will inhibit its corresponding "forward" detector. This detector in turn will be prevented from laterally inhibiting the "forward" detector in the adjacent retinal position.

This relationship may be expressed mathematically if one considers the responses of the depth detectors $\left(A_{1}, B_{1}, A_{2}\right.$, and $B_{2}$ in Figure 2$)$ to a certain stimulus energy. Assume that the depth response in the presence (D) or absence ( $\left.D^{\prime}\right)$ of lateral inhibition is given by the imbalance between front and back detectors,

$$
\mathrm{D}_{1}=\mathrm{A}_{1}-\mathrm{B}_{1} \quad \mathrm{D}_{1}^{\prime}=\mathrm{A}_{1}^{\prime}-\mathrm{B}_{1}^{\prime} .
$$

The responses of each detector are given when $A_{1}$ and $B_{2}$ are stimulated, and are shown here with only differential disparity inhibition on the left and with both types of inhibition on the right.

$$
\begin{array}{ll}
A_{1}=E-k B_{1} & A_{1}^{\prime}=E-k B_{1}^{\prime}-k A_{2}^{\prime} \\
B_{1}=-k A_{1} & B_{1}^{\prime}=-k A_{1}^{\prime}-k B_{2}^{\prime}
\end{array}
$$

where $\mathrm{k}$ is a constant determining the proportion of inhibition to excitation, which is assumed for simplicity to be the same for lateral and disparity inhibition. Now, by symmetry

$$
\mathrm{A}_{2}^{\prime}=\mathrm{B}_{1}^{\prime} \text { and } \mathrm{B}_{2}^{\prime}=\mathrm{A}_{1}^{\prime}
$$

Therefore,

$$
\begin{aligned}
A_{1} & =E+k^{2} A_{1} & A_{1}^{\prime} & =E+4 k^{2} A_{1}^{\prime} \\
& =\frac{E}{1-k^{2}} & & =\frac{E}{1-4 k^{2}}
\end{aligned}
$$

and

$$
B_{1}=\frac{-k E}{1-k^{2}} \quad B_{1}^{\prime}=\frac{-2 k E}{1-4 k^{2}} . \quad(6 a, b)
$$

Substituting in Equation 1 gives

$$
D_{1}=\frac{E(1+k)}{\left(1-k^{2}\right)} \quad D_{1}^{\prime}=\frac{E(1+2 k)}{\left(1-4 k^{2}\right)}
$$

But for $0<\mathrm{k}<1$, the inequalities $1+\mathrm{k}<1+2 \mathrm{k}$ and $1-k^{2}>1-4 k^{2}$ are valid. Therefore, in this range, it is always true that

$$
D_{1}<D_{1}^{\prime}
$$

Thus, according to this model, the depth perceived should be increased by the presence of lateral inhibition but decreased by the presence of disparity inhibition, and antiphase stereomovement should reduce or abolish stereomovement suppression. Figure 1 indicates that the antiphase stereomovement only abolishes the suppression below $0.5 \mathrm{~Hz}$. This suggests that differential disparity inhibition is only operative at these low frequencies. In this respect, it is similar to retinal lateral inhibition which is also restricted to low frequencies (Levinson, 1964; Kelly, 1969).

\section{FUSION OR DEPTH AS THE BASIS FOR STEREOMOVEMENT SUPPRESSION?}

Previous data (Tyler, 1971) showed that stereomovement suppression occurred only when the stimuli to the two eyes were perceived as fused. It is still ambiguous whether the suppression derives from the fusion or depth aspects of cortical disparity processing.

The horizontal/vertical anisotropy of the stereoscopic mechanism provides a convenient method of testing whether stereomovement suppression results from the fusional or depth processing. If the stimulus consists of vertical antiphase movement in horizontal lines, no depth is perceived. There is still a fusional range which is normally found to be about 6 arc min. If stereomovement suppression still occurs under these conditions, it cannot arise from the depth processing as such, but must be related to the processes of binocular fusion.

To make vertical movement observations, the apparatus was rotated by $90^{\circ}$ so that the test line was horizontal with a vertical sinusoidal movement in antiphase to the two eyes and a similar reference line with its movements in antiphase to the test line. The subject set monocular and binocular movement thresholds as before.

\section{Results}

The results (Figure 3) show a stereomovement suppression of the usual magnitude throughout the frequency range. At low frequencies, there is an interesting difference between vertical and horizontal novement sensitivity. Vertical stereomovement suppression increases to about one log unit, whereas 


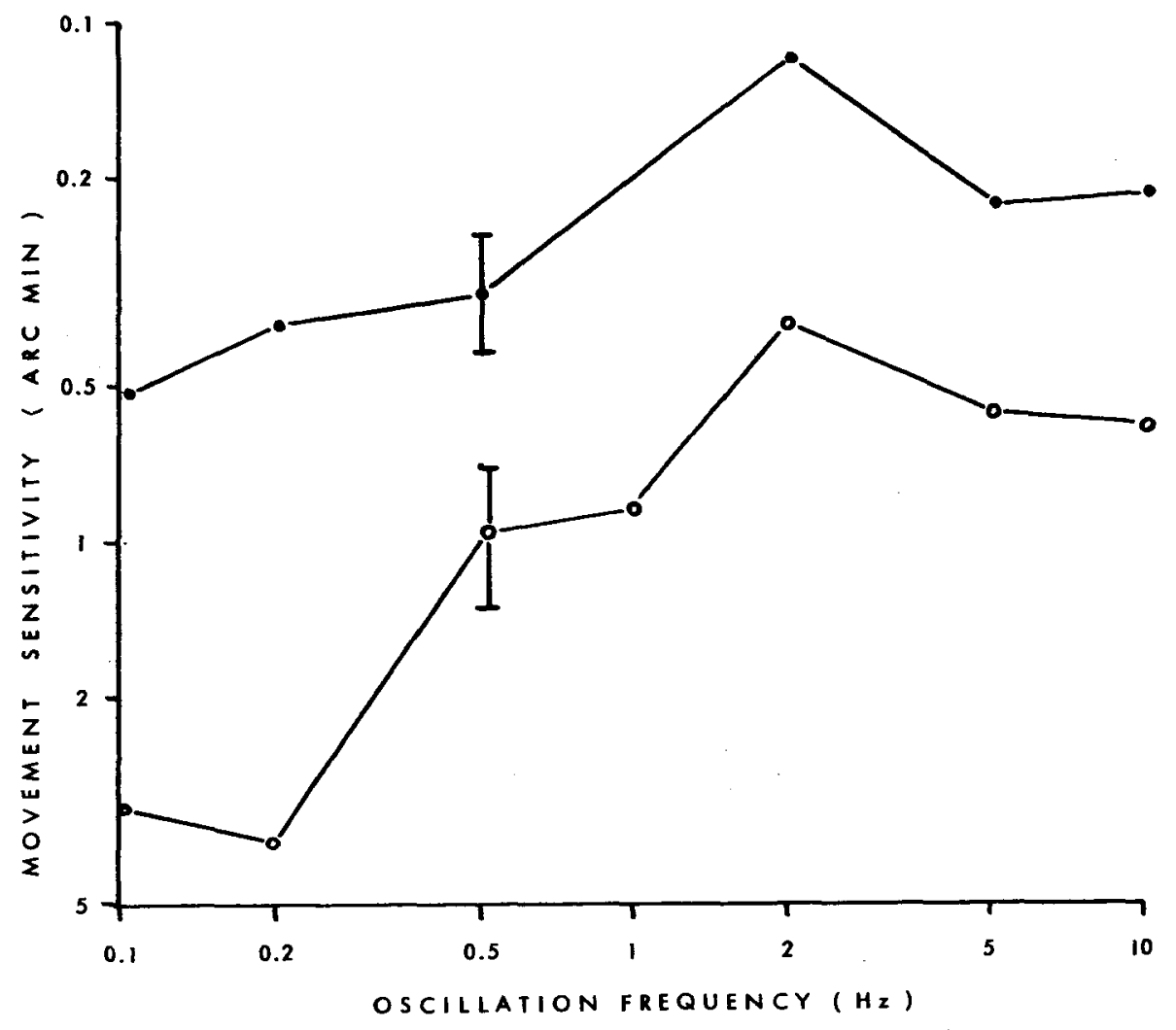

Figure 3. Stereomovement suppression for vertical movement of horizontal test lines (C.W.T.). Full chrcles, monocular sensitivity; open circles, binocular movement sensitivity.

horizontal stereomovement suppression (Figure 1) was abolished.

\section{Discussion}

Stereomovement suppression seems to be as marked for vertical as for horizontal movement (Figure 3), suggesting that suppression arises from processing for fusion, which occurs in both horizontal and vertical meridians, rather than for depth, which occurs only in the horizontal meridian. In this connection, Barlow, Blakemore, and Pettigrew (1967) make clear the distinction between the region of binocular facilitation (where the binocular response is greater than the sum of the two monocular responses of a neuron) and the absolute binocular disparity of the receptive fields of a cortical cell. For example, a neuron may show a maximum response at $6^{\circ}$ absolute disparity of the stimuli to the two eyes and yet have a range of facilitation of less than $1^{\circ}$ about this disparity. Outside the range of facilitation, the binocular response is indistinguishable from the monocular response. Fusion is probably some function of the range of facilitation across the population of cells, whereas depth is presumably derived from the absolute disparity. In this way, both perceptual limits may be related to the response of a single cortical neuron, although the limits have different properties.
Since the vertical disparities are not processed for depth, there is no reason to suppose that differential disparity inhibition would be present in the vertical direction. The presence of strong vertical stereomovement suppression at low frequencies (Figure 3) therefore supports the hypothesis that the low-frequency abolition of horizontal stereomovement suppression (Figure 1) results from lateral disparity inhibition, as suggested in Section I.

\section{STEREOMOVEMENT AND RETINAL ECCENTRICITY}

It is important to know whether the stereomovement suppression extends over the visual field or is limited, for example, to the fovea. Sensitivity to monocular and stereoscopic depth movement was therefore measured as a function of retinal eccentricity.

Informal observation suggested that there was a region in which antiphase stimulus movement was apparent without any impression of movement in depth. Such a region was hardly noticeable in the foveal study. In addition to the monocular movement threshold, two criteria were therefore adopted for the stereosopic movement threshold: (a) threshold of antiphase binocular movement, and (b) threshold of stereomovement in depth. 


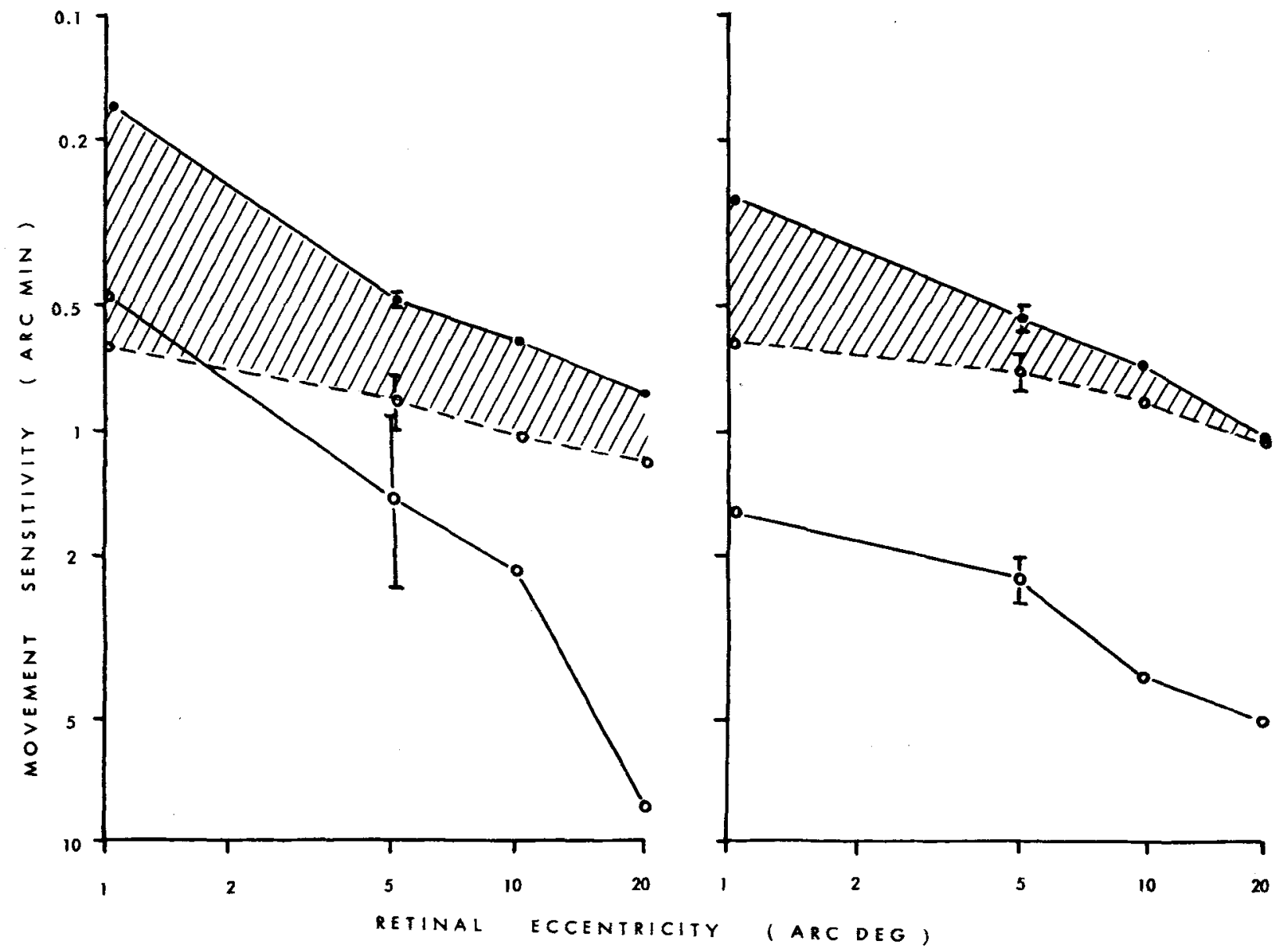

Figure 4. Stereomovement suppreasion in the periphery (C.W.T.). Full eircles, full Ine-monocular movement sensitivity; open circles, dashed line- - binocular movement sensitivity; open circles, full line-depth movement sensitivity. Stereomovement suppreasion indicated by cross-hatehed region. Left-hand graph, 0.5 Hz; right-hand graph, 5 Hz.

\section{Method}

The experimental procedure was the same as for Experiment $I$. except that a stationary reference line was used. The experiment was performed at two oscillation frequencies $(0.5$ and $5 \mathrm{~Hz})$, where there was little difference between sensitivities for stationary or oscillating reference. A binocular fixation point was provided at angular distances of $1,5,10$, and 20 arc deg from the stimulus. The stereoscopic distance of the fixation point was adjusted so that the stimulus appeared optimally fused when stationary and thus lay on the horopter.

\section{Results}

The results for Subject C.W.T. (Figure 4) show that sensitivity with all three criteria was reduced in peripheral viewing. Monocular movement sensitivity (full circles) was not reduced to the same extent as in a previous study (Tyler \& Torres, 1972), but the observation conditions are different. The difference between antiphase movement sensitivity (dashes) and monocular sensitivity represents the stereomovement suppression effect (cross-hatched area). At both frequencies, this suppression is large at the fovea, in agreement with the data of Figure 1 , and dwindles in peripheral observation. Finally, stereomovement sensitivity (open circles) diverges markedly from antiphase movement sensitivity, in the periphery at the low frequency and in all retinal locations at the high frequency,

\section{Discussion}

The data of Figure 4 may be regarded as a reduction in both depth sensitivity and stereomovement suppression with increasing eccentricity. Classical stereoacuity also declines with increasing eccentricity. A simple model for these results could be a reduction in the proportion of binocular cells showing facilitation to those showing summation with increasing eccentricity. (Pettigrew, Nikara, \& Bishop, 1968). A summating cell should detect antiphase movement just as well as monocular movement (assuming that there is no inhibition within the nonpreferred direction). Summation of this type could be the predominant processing in the periphery. On the other hand, a facilitating cell should show stereomovement suppression and processing of disparity for depth. If facilitating cells increased towards the fovea, one would expect a corresponding 
increase in both stereomovement suppression and depth movement sensitivity, as is evident in the data (Figure 4). Current physiological data are not sufficiently extensive to resolve whether such a distribution of summatory and facilitatory cells occurs anatomically.

\section{ADDENDUM}

Regan and Beverley (1973a) have recently reported stereomovement thresholds with somewhat different characteristics from those of Tyler (1971). In stereonormal subjects, lateral movement thresholds were up to twice as high as stereomovement thresholds in the range of $\pm 10^{\prime}$ disparity from the fixation plane at $0.1 \mathrm{~Hz}$ sinusoidal oscillation. These effects disappeared by about $1 \mathrm{~Hz}$. Furthermore, in experiments using slightly different frequencies of oscillation of random-dot stimuli in the two eyes, in which the depth oscillation fluctuates at the difference frequency, the same authors obtain related results (Regan \& Beverly, 1973b). For "almost all situations sensitivity to depth oscillations was lower than sensitivity to sideways oscillations." Similarly, Beverly and Regan (1974) report data thresholds for pulsed changes in disparity in which disparity sensitivity is poorer by a factor of 3 than movement sensitivity.

The data of the present study suggest a hypothesis which can simultaneously explain the form of the stereomovement thresholds obtained by Regan and Beverley (1973a) and the discrepancy with those of Tyler (1971) and the present study. The hypothesis suggests that absolute stereomovement sensitivity is low, but that for stereomovement relative to a fixed reference point, adjacent both laterally and in disparity to the mean position of the test line, sensitivity can be greatly increased. This hypothesis encompasses the experimental results as follows:

(a) Stereomovement suppression was found by Tyler (1971) because the fixation line was not close enough to act as an adequate reference, hence absolute stereomovement sensitivity was effectively being measured.

(b) Regan and Beverley (1973a, b) found enhanced stereomovement sensitivity when the random-dot fixation field could be used as a disparity reference, i.e., within $\pm 10^{\prime}$ disparity from the test line. Stereomovement suppression was observed when the relative disparity was too great for the fixation field to be effective as a disparity reference.

(c) If the absolute/relative disparity distinction is valid, we would predict that when the stimulus approximated an absolute disparity situation, viz, at large disparities from the plane of fixation, sensitivity should decrease with increasing frequency, as found by Tyler (1971). On the other hand, at small disparities from fixation, the viability of the relative disparity cue should tend to make sensitivity independent of frequency. Both predictions are clearly borne out in Regan and Beverley's (1973a) data.

In conclusion, I suggest that the apparent discrepancy between the two sets of data is accounted for by the presence or absence of relative disparity cues. Presumably absolute disparity sensitivity is determined by the characteristics of individual disparity detectors and their interactions in the disparity domain, whereas relative disparity sensitivity is determined by interactions between disparity detectors laterally along the cyclopean retina.

\section{REFERENCES}

Barlow, H. B., Blakemore, C., \& Pettigrew, J. D. The neural mechanism of depth discrimination. Journal of Physiology, $1967,193,327-342$.

Beverley, K. I., \& Regan, D. Visual sensitivity to disparity pulses. Vision Research, 1974, 14, 357-361.

KELLY, D. H. Flickering patterns and lateral inhibition. Journal of the Optical Society of America, 1969, 59, 1361-1370.

LeVINson, J. Z. Non-linear and spatial effects in the perception of flicker. Documenta Ophthalmalogica, 1964, 18, 36-55.

Pettigrew, J. D., Nikara, T., \& Bishop, P. O. Binocular interaction on single units in cat striate cortex. Experimental Brain Research, 1968, 6, 391-410.

Regan, D., \& Beverley, K. I. Some dynamic features of depth perception. Vision Research, 1973, 13, 2369-2379. (a)

Regan, D., \& Beverley, K. I. The dissociation of sideways movements from movements in depth: Psychophysics. Vision Research, 1973, 13, 2403-2415. (b)

Tyler, C. W. Stereoscopic depth movement: Two eyes less sensitive than one. Science, 1971, 174, 958-961.

TYLER, C. W., \& ToRres, J. Frequency response characteristics for sinusoidal movement in the fovea and periphery. Perception \& Psychophysics, 1972, 12, 232-236.

Yoshida, T., \& Watanabe, A. Analysis of interaction between accommodation and vergence feed back control systems of human eyes. Bulletin NHK Broadcasting Science Research Laboratory, $1969,3,72-80$.

Zuber, B. L., \& Stark, L. Dynamical characteristics of the fusional vergence eye-movement system. IEEE Transactions on Systems, Science, and Cybernetics, 1968. SSC-4, 72-79.

(Received for publication June 10, 1974; revision received October 11,1974 .) 